Ann. Biol. anim. Bioch. Biophys., I964, 4 (2), I57-I6r

\title{
MARQUAGE DES CELLULES GERMINALES DU BÉLIER ET DU TAUREAU PAR INJEGTION DE THYMIDINE TRITIÉE DANS L'ARTËRE SPERMATIQUE
}

\author{
Marie-Thérèse HOCII REAU, M. COUROT et R. OR'LAVANT \\ avec la collaboration technique de Claire Burt, Liliane Borvineau et $\mathrm{Y}$. de Fontaubert \\ Laboratoire de Physiologie de la Reproduction, \\ Centre national de Recherches zootechniques, Jouy-en-Josas (Seine-et-Oise)
}

\section{SOMMAIRE}

L'injection de très faibles doses de thymidine tritiée $(500 \mu \mathrm{c})$ dans l'artère spermatique du bélier et du taureau permet un marquage des cellules germinales suffisant pour être détecté par autoradiographie du testicule.

La durée du cycle de l'épithélium séminifère a été vérifiée dans les deux espèces étudiées : $10,4 \mathrm{j}$ chez le bélier et $\mathbf{I} 3,5 \mathrm{j}$ chez le taureau. Cette technique peut être généralisée pour l'étude des substances agissant sur la spermatogenèse dans ces deux espèces.

\section{IN'TRODUC'TION}

La thymidine tritiée a été utilisée avec succès dans l'étude de la spermatogenèse des Mammifères (Clekmont et al., I959; CLERMONT, I962 ; HelleER et al., I962 ; Monesi, I 962 ; Descin et ORTAVANT, I963). I,es autoradiographies effectuées sur du tissu germinal marqué par le tritium permettent un repérage très précis des cellules germinales marquées.

Chez les petits Mammifères, l'élément radioactif est injecté par voie sous-cutanée à raison de $\mathrm{I} \mu \mathrm{c} / \mathrm{g}$ de poids vif. Cette méthode ne peut être appliquée chez les gros animaux car elle entraînerait l'injection de trop grandes quantités de radioéléments. C'est pourquoi nous avons cherché à mettre au point une technique d'injection directe, dans l'artère irriguant l'organe à étudier, permettant ainsi de réduire considérablement la dose administrée. 


\section{MATÉRIEI, ETT MÉTHODES}

Nous avons utilisé deux béliers adultes (de race Ile-de-France) et six taureaux de $\mathrm{r} 8$ mois à 5 ans (de race Française Frisonne Pie noire, FFPN, ou croisés, FFPN $\times$ Normande).

\section{I. - Technique opératoire}

Après administration de chlorpromazine $(50 \mathrm{mg} / \mathrm{I} 00 \mathrm{~kg}$ de poids vif), l'anesthésie a été obtenue par l'injection intraveineuse de 30 à $50 \mathrm{ml}$ d'un mélange de barbituriques (1) chez le bélier, et de 250 à $900 \mathrm{ml}$ d'Équithésine ( $\left.{ }^{2}\right)$ chez le taureau.

- Chez le bélier, après ouverture longitudinale de la paroi cutanée du scrotum, de la tunique fibreuse et de la tunique vaginale, le cordon testiculaire est isolé. Le trocard d'injection est introduit à travers le complexe veineux dans l'artère testiculaire, juste avant les premières circonvolutions du cône vasculaire (fig. I) ; $500 \mu \mathrm{c}$ de thymidine tritiée diluées dans $50 \mathrm{ml}$ de sérum physiologique sont injectées.

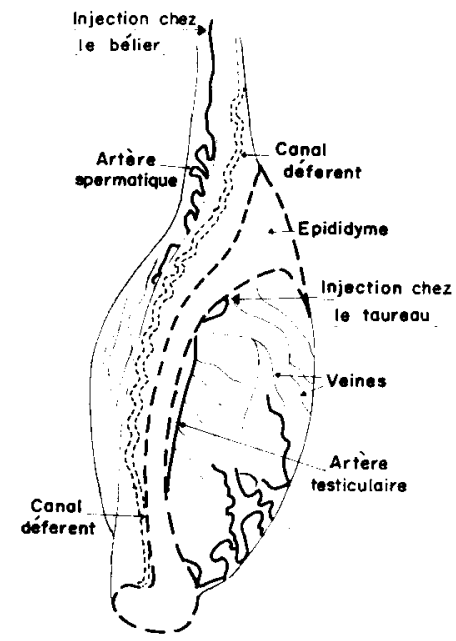

FıG. I. - Schéma de la vascularisation du: estucule montrant', es lieux où ont été pratiquées les injections chez le bélier et chez le taureau

- Chez le taureau, l'injection dans l'artère spermatique au niveau du cordon est souvent suivie d'un collapsus artériel plus ou moins important. Nous avons essayé une voie d'injection plus basse, juste à la sortie du cône vasculaire, alors que l'artère spermatique n'est pas encore divisée et chemine dans l'épaisseur de la tunique albuginée, sous la tête distale de l'épididyme (fig. r). Nous avons injecté $600 \mu \mathrm{l}$ de thymidine tritiée diluées dans ro $\mathrm{ml}$ de sérum physiologique

Dans les deux espèces, la durée de l'injection est assez longue, de l'ordre de dix minutes. La petite hémorragie provoquée par le passage du trocard est arrêtée par une électrocoagulation douce chez le bélier et avec de la thrombase chez le taureau.

Les animaux ont été castrés à des temps différents après l'injection. $5 \circ \mathrm{ml}$.

(1) Formule du mélange $=$ Nembutal $5 \mathrm{ml}$, Pentothal I g, Atropine $5 \mathrm{mg}$, eau : quantité suffisante pour

$\left({ }^{2}\right)$ Formule de l'Équithésine $=$ Hydrate de chloral $42,5 \mathrm{~g}$, Nembutal 9,75 g, Sulfate de magnésium 21,25 g, solution aqueuse de propylène glycol à $35 \mathrm{p}$. 100 et Éthanol $5 \mathrm{p}$. 100, quantité suffisante pour I litre. 


\section{2. - Méthodes autoradiographiques}

Les fragments de testicule prélevés sont fixés au Bouin-Hollande, déshydratés et inclus dans la paraffine. Les coupes de 1o $\mu$ d'épaisseur, après coloration au Feulgen sont recouvertes d'émulsion Ilford $\mathrm{K}_{5}$ coulable ou Kodak ARIO pelliculable (KOPRIwA et LEBLOND, I962), exposées de 30 à 45 jours et révélées selon les procédés classiques, à l'aide d'un révélateur à l'amidol (4 minutes pour l'émulsion coulable, 8 minutes pour l'émulsion pelliculable).

\section{RÉSULTATS PRÉLIMINAIRES}

Dans une première expérience, nous avons vérifié, en injectant un colorant vital qu'il $\mathrm{y}$ avait une répartition homogène de celui-ci dans tout le testicule. Chez le taureau, celle-ci est meilleure lorsque l'injection a lieu dans l'artère spermatique au niveau de l'albuginée.

Dans les deux espèces, une demi-heure après l'injection de thymidine tritiée, les spermactoytes I sont marqués au stade préleptotène (fig. 2). C'est donc le stade ultime auquel du matériel radioactif s'incorpore dans les cellules germinales. Ainsi nous pouvons suivre l'évolution de ces cellules marquées.

Chez le bélier, 27 jours après 1'opération, les spermatozoïdes marqués, issus des spermatocytes en synthèse au moment de l'injection, bordent la lumière des tubes séminifères (stade 7 , suivant ORTAVANT, I958). Chez le taureau, I9 jours après l'injection, les spermatocytes marqués sont au stade des divisions réductionnelles (stade 4).

Nous pouvons ainsi calculer la durée du cycle de l'épithélium séminifère dans les deux espèces ; elle est de 13,5 jours chez le taureau et de 10,4 jours chez le bélier. Ceci confirme les résultats obtenus après injection de ${ }^{32} \mathrm{P}$ par ORTAVANT (r956) et ORGEBIN-CRIST (r962).

En outre, il est possible d'étudier l'évolution des spermatogonies marquées au moment de l'injection : en effet, chez le taureau castré une heure après l'injection, les spermatogonies A sont marquées en fin de stade I et au stade 2, les spermatogonies intermédiaires aux stades 3 et 4 et enfin les spermatogonies B aux stades 7 et 8 . Chacune de ces catégories de spermatogonies évolue ensuite pour donner naissance à des vagues successives de cellules marquées.

Il est cependant à noter que dans certains cas l'opération chirurgicale peut entraîner une diminution de l'activité spermatogénétique.

\section{CONCLUSION}

Cette technique d'injection directe dans l'artère irriguant le testicule permet d'utiliser des doses de radioéléments qui, pour un taureau, ne sont pas plus élevées que celles utilisées pour marquer les cellules germinales chez un rat. Elle offre la possibilité d'étudier 1'action spécifique des facteurs qui contrôlent la spermatogenèse chez les animaux domestiques. 


\section{SUMMARY}

\section{LABELLING OF GERM CELLS IN THE RAM AND IN THE BULL BY INJECTION OF TRITIATED THYMIDINE IN THE SPERMATIC ARTERY}

A technique for the injection of tritiated thymidine into the spermatic artery has been developed for the ram and the bull. The site of injection in the ram is in front of the vascular cone, and in the bull in the artery penetrating the tunica albuginea. Small doses of about $500 \mu \mathrm{c}$ are sufficient for a labelling of the germ cells detectable by autoradiography.

Therefore, it was possible to confirm the duration of the seminiferous epithelium cycle, ro,4 days in the ram and ${ }_{13}, 5$ days in the bull. This injection technique may be utilized for the study of the action of factors which control spermatogenesis in the domestic animals.

\section{RÉFÉRENCES BIBLIOGRAPHIQUES}

Clermont Y., 1962, Quantitative analysis of spermatogenesis of the rat : a revised model for the renewa of spermatogonia. Amer. J. Anat., 111, I I I-I 29 .

Clermont Y., Leblond C. P., Messier B., i959. Durée du cycle de l'épithélium séminal du rat. Arch. Anat. Micr. Morph. exp., 48 bis, 37-54.

Desclin J., ORTAVANT R., I963. Influence des hormones gonadotropes sur la durée des processus spermatogénétiques chez le rat. Ann. Biol. anim. Bioch. Biophys., 3, 329-342.

Heller C. G., Matson L. J., Moore D. J., Clermont Y., ig62. Rate of spermatogenesis in man determined by incorporating tritiated thymidine into testes, in CARLSON W. D., GASSNER F. X., r963. - Effects of Ionizing Radiation on the Reproductive system, 263-267, Pergamon Press.

KopRIWA B. M., LEBLOND C. P., I962. Improvments in the coating technique of radioautography. Histochem. Cytochem., 10, 269-284.

MONESI C. V., I962. Autoradiographic study of DNA synthesis and the cell cycle in spermatogonia and spermatocytes of mouse testis using tritiated thymidine. J. Cell Biol., 14, I-18.

Orgebin-Crist M. C., 1962. Recherches expérimentales sur la durée de passage des spermatozoïdes dans l'épididyme du taureau. Ann. Biol. anim. Bioch. Biophys., 2, $5^{\mathrm{I}-108 .}$

Ortavant R., 1956. Autoradiographie des cellules germinales du testicule de bélier. Durée des phénomènes spermatogénétiques. Arch. Anat. Micr. Morph. exp., 45, 1-ro.

Ortavant R., I958. Le cycle spermatogénétique chez le bélier. Thèse Doct. Paris, i27 p., éd., 1959, Ann. Zootech., 8, I83-244 et 27 I-322. 

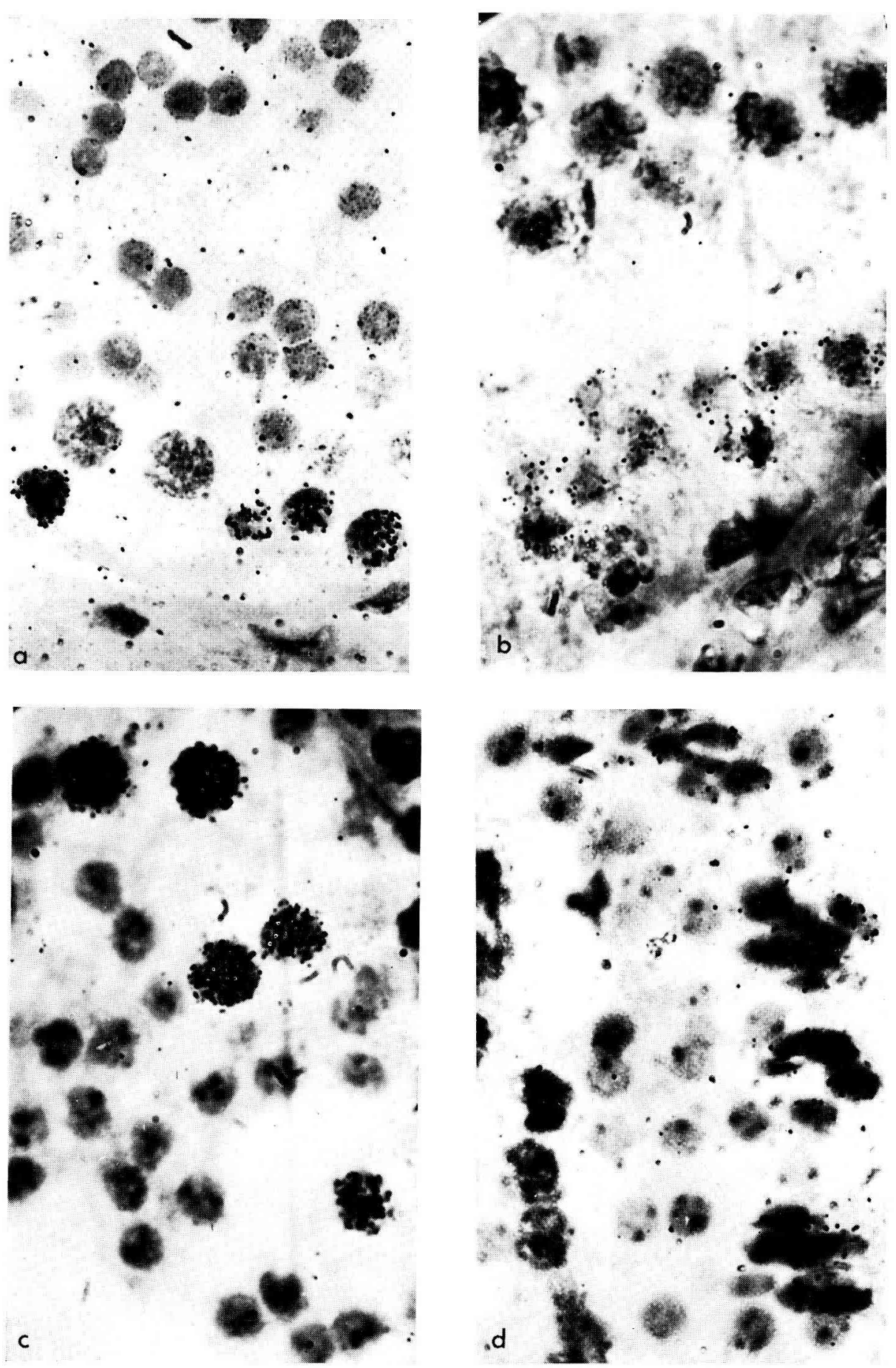

FIG. 2. - Autoradiographies testiculaires apies injection de inymiäine tritiee

- $a$ et $b$, bélier et taureau, $30 \mathrm{mn}$ : les spermatocytes I au stade préleptotène sont marqués (stade I) - c, taureau, $13 \mathrm{j} 6 \mathrm{~h}$ : les spermatocytes I en fin de stade pachytìne sont marqués (stade $\mathrm{I}$ );

- $d$, bélier, 27 j : les sjermatozoïdes du stade 7 sont marqués. 